\title{
Génétique moléculaire des dysgénésies testiculaires
}

\author{
Ch. SULTAN*, **, S. LUMBROSO*, J.M. LOBACCARO* \\ *Unité de Biochimie Endocrinienne du Développement et de la Reproduction, Hôpital Lapey- \\ ronie et INSERM U58, Montpellier - **Endocrino-Gynécologie Pédiatriques, Service de \\ Pédiatrie 1, Hôpital Arnaud de Villeneuve, Montpellier
}

\section{RESUME}

La détermination testiculaire, première étape de la différenciation sexuelle masculine, est sous le contrôle de facteurs génétiques. Le rôle clé du gène SRY dans ces mécanismes est maintenant bien établi. Cependant de nombreux arguments, cliniques et génétiques, plaident en faveur de l'intervention d'autres gènes en aval ou en amont de SRY.

Ainsi, la plupart des hommes $46, \mathrm{XX}$ ont hérité, par translocation, du gène SRY, responsable de la différenciation des gonades dans le sens testiculaire, mais dans $10 \%$ des cas le gène SRY n'est pas retrouvé. De même, si l'analyse de femmes 46,XY a participé à l'identification du gène SRY, $80 \%$ des cas de dysgénésies gonadiques ne sont pas expliqués par une anomalie de SRY.

Un certain nombre de situations cliniques permettent de suspecter le rôle des autosomes (chromosomes 1, 9, 10 17...) et du chromosome $X$ dans la pathologie de la détermination sexuelle. Les travaux les plus avancés concernent le facteur TDF-X, qui agirait comme un represseur de la détermination testiculaire.

Au total, les mécanismes moléculaires de la détermination sexuelle apparaissent extrêmement complexes, faisant intervenir probablement plusieurs gènes en une cascades de réactions.

Mots clés : Détermination sexuelle, SRY, mâles XX, dysgénésies testiculaires.

\section{INTRODUCTION}

La différenciation sexuelle représente l'ensemble des phénomènes qui participent à l'établissement du dimorphisme sexucl. Ce processus comporte deux étapes : la détermination du sexe, c'est à dire l'orientation de la gonade primitive qui est gouvernée par l'équipement génétique des chromosomes sexuels et la différenciation sexuelle proprement dite qui correspond à la mise en place des tractus génitaux interne et externe, étape qui relève de l'action de facteurs hormonaux sécrétés par la gonade foetale: hormone anti-müllérienne, sécrétée par les cellules de Sertoli et androgènes, sécrétés par les cellules de Leydig [9].

Si le rôle du chromosome $Y$ dans la détermination testiculaire est connu depuis longtemps, la localisation exacte du facteur de détermination testiculaire (TDF, Testis Determining Factor) est plus récente. L'analyse de femmes XY avec dysgénésie gonadique pure présentant des mutations du gène SRY [2,6] et d'hommes XX ayant hérité, par translocation de matériel Y ont permis de localiser ce gène TDF dans une région de $35 \mathrm{~kb}$, adjacente à la région pseudo-autosomique sur le bras court du chromosome $Y$. Le gène identifié dans cette région a été appelé SRY pour Sex-determining Region, Y chromosome [17].

Cependant, de nombreux arguments cliniques et expérimentaux démontrent que si le gène SRY exerce un rôle clé dans la détermination testiculaire, celle-ci requiert vraisemblablement l'action d'autres gènes 
(autosomiques ou liés à l'X) intervenant en amont ou en aval de SRY.

Les anomalies de la différenciation testiculaire recouvrent un ensemble hétérogène de situations cliniques, biologiques et génétiques. Elles constituent un modèle privilégié pour appréhender les mécanismes extrêmement complexes de la détermination sexuelle.

\section{Les hommes $46, \mathrm{XX}$}

Si l'identification du gène SRY a bénéficié de l'analyse des individus présentant une réversion sexuelle du fait d'une translocation de TDF de l'Y sur l'X, les études d'un plus grand nombre de cas ont souligné l'hétérogénéité clinique et génétique de ces situations. Trois groupes peuvent être distingués :

Les mâles XX sans ambiguité sexuelle présentent des organes génitaux internes et externes masculins normaux. Les testicules sont le plus souvent atrophiques, et l'azoospermie est constante. Sur le plan génétique, le caryotype est pur, 46,XX. Dans la grande majorité des cas le gène SRY est présent et normal. Chez environ $10 \%$ des patients le SRY n'est cependant pas retrouvé. Deux hypothèses peuvent expliquer cette situation paradoxale d'une différenciation sexuelle masculine normale en l'absence du gène SRY. L'existence d'une mosaïque indétectable ne peut être formellement écartée, mais semble peu probable étant donné la sensibilité des techniques de biologie moléculaire (PCR) utilisée pour rechercher le gène SRY. La possibilité d'une mutation d'un gène intervenant en aval de SRY apparaît plus vraisemblable. Ainsi l'hypothèse du gène TDF-X, que nous développons plus loin, peut expliquer ces réversions sexuelles XX sans gène SRY.

L'hypothèse d'autres gènes que SRY nécessaires à une différenciation sexuelle normale, et en particulier de gènes de maintenance testiculaire, est corroborée par l'absence de cellules germinales chez les mâles XX qui présentent une différenciation normale des tractus génitaux.

Les mâles XX avec ambiguité des organes génitaux externes de degré variable, présentent des dérivés müllériens souvent associés aux organes génitaux internes mâles. Les gonades sont de structure testiculaire. Le gène SRY est le plus souvent absent $(90 \%)$, ce qui souligne encore une fois le rôle d'autres gènes que SRY dans la détermination gonadique. Dans $10 \%$ des cas, le SRY est présent et normal. Un effet de positionnement du matériel Y transloqué pourrait expliquer un dysfonctionnement du gène SRY et donc l'anomalie de la différenciation testiculaire.

Les hermaphrodismes vrais $46, \mathrm{XX}$ sont proches des mâles XX avec ambiguïté génitale ; mais l'ambiguïté est plus marquée conduisant, dans $2 / 3$ des cas, à un élevage dans le sens féminin, et surtout, il y a présence au niveau des gonades de tissu testiculaire et de tissu ovarien à la fois. Le gène SRY est le plus souvent absent et les mosaïques ne sont pas rares. Mâles XX et hermaphrodismes vrais peuvent être considérés comme appartenant à un large spectre clinique, allant selon un continuum, des mâles XX sans ambiguïté aux hermaphrodismes vrais avec phénotype féminin presque parfait.

\section{Les dysgénésies gonadiques $46, X Y$}

Il faut distinguer les dysgénésies gonadiques mixtes, avec mosaiqque et les dysgénésies $46, \mathrm{XY}$ sans mosaïque.

\section{a) Les dysgénésies gonadiques mixtes}

(DGM) recouvrent un groupe hétérogène d'anomalies chromosomiques, gonadiques et phénotypiques. Mendez et al ont récemment rapporté l'analyse clinique, endocrinienne et cytogénétique de 16 patients porteurs de DGM [13]. l'ambiguité génitale, constante mais de degré variable, associe le plus souvent un micropénis, un hypospadias et une ectopie testiculaire. Les gonades et les tractus génitaux internes sont mixtes. D'un côté on observe une structure testiculaire avec dérivés wolffiens (epididyme, déférent) et rarement quelques 
dérivés mülleriens. De l'autre côté les gonades sont réduites à des bandelettes fibreuses et le tractus comporte des dérivés mülleriens associés dans $1 / 3$ des cas à des dérivés wolffiens. Des stigmates turnériens sont fréquemment retrouvés : ceux-ci concernent essentiellement la face et les doigts, mais la croissance staturale est normale.

Le bilan endocrinien met en évidence, après la puberté des taux élevés de FSH et LH, avec une testostérone normale ou basse, avant la puberté des taux normaux de gonadotrophines avec une testostérone normale.

Le caryotype montre une mosaíque 46 , $\mathrm{X} / 46, \mathrm{XY}$ dans $90 \%$ des cas. Il est intéressant de noter deux observations de caryotype $46, \mathrm{XY}$, sans mosaïcisme. Enfin, le risque de gonadoblastome est élevé ( 3 cas sur 16). Les études en génétique moléculaires n'ont révélé une anomalie du gène SRY que dans $25 \%$ des cas.

\section{b) Les dysgénésies gonadiques 46,XY} peuvent être définies par l'absence ou le défaut de détermination testiculaire sans mosaïcisme. On distingue les dysgénésies gonadiques complètes et les dysgénésies gonadiques partielles.

\section{Les dysgénésies gonadiques complètes.}

- Les dysgénésies gonadiques pures (syndrome de Swyer).

Sur le plan clinique, cette affection se caractérise par un tableau d'impubérisme avec aménorrhée primaire et parfois des signes de la série turnérienne, en particulier une petite taille. Les organes génitaux externes sont féminins, sans ambiguïté. De même, le tractus génital interne est constitué exclusivement de dérivés müllériens. Les gonades sont réduites à l'état de bandelettes fibreuses avec dans de rares cas quelques follicules primaires. Le caryotype est $46, \mathrm{XY}$.

La découverte de mutations du gène SRY dans des cas de DGP a été un des arguments essentiels qui ont permis de considérer que SRY était le gène de détermination testiculaire $[2,6]$. Depuis, plusieurs autres mutations de novo de SRY ont été rapportées $[4,10,19]$. Les mutations ponctuelles décrites à ce jour siègent toutes au niveau de la boîte HMG, région très conservée du gène SRY. Des délétions ont également été mises en évidence en $3^{\prime}$ et $5^{\prime}$ du gène, qui modifient probablement des éléments de régulation du gène [11].

\section{- Le syndrome de Drash.}

Ce syndrome est caractérisé par l'association d'une tumeur de Wilms, d'une ambiguité génitale et d'une insuffisance rénale due à une glomérulonéphrite spécifique. L’origine génétique de ce syndrome a pu être déterminée grâce à la découverte récente de mutations ponctuelles du gène suppresseur de la tumeur de Wilms, WT1 [15]. Aucune anomalie de SRY n'est retrouvée dans ce syndrome [16]ce qui a conduit à envisager que le gène WT1 agirait en aval de SRY au cours de la différenciation sexuelle. Cette hypothèse doit cependant être écartée, car il est maintenant démontré que l'expression $\mathrm{du}$ gène WT1 débute, au cours de la vie fotale, avant celle du gène SRY.

- Le syndrome de Frasier.

Le syndrome de Frasier se distingue du syndrome de Drash par l'absence de Tumeur de Wilms et d'ambiguïté génitale, puisque les patientes présentent un phénotype féminin normal. L'origine moléculaire du syndrome de Frasier semble être différente de celle du syndrome de Drash. En effet, aucune anomalie du gène WT1 n'a été retrouvée dans une étude récente portant sur 3 cas [16]. De plus, aucune anomalie de SRY n'est retrouvée dans ce syndrome [3].

- L'anorchidie bilatérale congénitale.

L'anorchidie bilatérale congénitale, dont l'incidence est d'environ 1/20000 naissances masculines, est définie par l'absence complète de tissu testiculaire chez des individus $46, \mathrm{XY}$ de phénotype masculin normal : les organes génitaux internes et externes sont de type masculin sans ambiguïté. L'absence de structures müllériennes et la différenciation normale des dérivés wolffiens chez ces 
patients, suppose que l'hormone anti-müllériene (HAM) et les androgènes ont été sécrétés par le testicule fotal, et donc que cellules de Sertoli et de Leydig ont été présentes au cours de la période critique de différenciation sexuelle. Lobaccaro et al. [8] dans une étude récente portant sur 4 patients n'ont pas retrouvé d'anomalie du gène $S R Y$. Une origine génétique à ce syndrome ne peut cependant pas être écartée car des modes de transmission autosomiques ou liés à l'X ont été rapportés [12]. On ne peut cependant pas exclure une origine mécanique à cette affection : une torsion du testicule fotal conduirait à une destruction du testicule in utero via un défaut de vascularisation [5].

\section{Les dysgénésies gonadiques partielles.}

Les dysgénésies gonadiques partielles (DGP) sont caractérisées par l'association d'une ambiguïté génitale, de structures mülleriennes et wolffiennes et de gonades dysgénésiques. Les gonades comportent le plus souvent des ébauches de structures testiculaires (tubules séminiferes désorganisés) associées à du stroma ovarien. Le caryotype est $46, \mathrm{XY}$ sans mosaïcisme. Dans ces formes de dysgénésie gonadique aucune mutation du gène SRY n'a été retrouvée. Il est intéressant de noter que certaines formes de DGP sont associées à une transmission héréditaire liées à $1{ }^{\prime} \mathrm{X}$.

\section{Les autres gènes impliqués dans le processus de détermination sexuelle}

Un certain nombre de situations cliniques ne peuvent être expliquées uniquement par une anomalie de SRY [20] et permettent d'envisager l'intervention d'autres gènes, autosomiques ou liés à l'X, en amont ou en aval de SRY.

a) $80 \%$ des dysgénésies gonadiques ne relèvent pas d'une anomalie du gène SRY.

L'analyse moléculaire du gène SRY dans les dysgénésies gonadiques ne retrouve de mutation que dans environ 20 à $30 \%$ des cas.
Une étude récente sur 14 cas de DGP 46,XY montre l'importance d'une étude histologique très minutieuse en matière de dysgénésies gonadiques [19] : en l'absence de mutation de SRY l'examen histologique retrouve toujours des structures différenciées au niveau des gonades dysgénésiques (rete testis, tubules, cellules germinales). En cas de mutation de SRY, on ne retrouve que des bandelettes fibreuses sans aucun structure différenciée.

Il reste néanmoins à expliquer la reversion sexuelle chez ces sujets sans mutation de SRY. Il pourrait s'agir d'anomalie de séquences régulatrices de l'expression de SRY, ou plus probablement de l'intervention d'un deuxième gène.

\section{b) La mutation de SRY peut être retrou- vée chez le père ou les frères de patients.}

Quelques cas ont en effet rapportés de patients présentant une dysgénésie gonadique avec mutation de SRY et dont la même mutation est retrouvée chez le père ou chez le frère $[7,20]$. Si le gène SRY était seul responsable de la détermination testiculaire, comment expliquer l'absence de survenue de dysgénésie gonadique chez ces sujets apparentés?

c) Environ $10 \%$ des hommes $\mathrm{XX}$ sans ambiguïté sexuelle ne possèdent pas le gène SRY

\section{LES GENES CANDIDATS}

Un certain nombre de situations cliniques associant reversion sexuelle et divers syndromes malformatifs ou d'observation de délétions chromosomiques associées à une réversion sexuelle permettent de suspecter le rôle des autosomes et du chromosome $\mathrm{X}$ dans la pathologie de la détermination sexuelle.

\section{a) Le chromosome 17.}

Une étude récente [18] de cas de dysplasie campomélique associée à une réversion sexuelle, a localisé un locus autosomique lié à cette affection sur le chromosome 17 en position 17q24.3-q25.1. 


\section{b) Le chromosome 9.}

Bennet et al [1] rapportent un cas de réversion sexuelle associée à une délétion du bras court du chromosome 9 .

\section{c) Le chromosome 10.}

Wilkie et al [21] ont récemment décrit deux cas de dysgénésies gonadiques complète et partielle associées à une délétion terminale du bras long du chromosome 10 .

\section{d) Le chromosome 1.}

Des études sur la détermination testiculaire chez la souris ont permis d'identifier un locus autosomique (Tda1) sur le chromosome 4 (homologue du chromosome 1 humain). Le gène Tda1 interviendrait en aval de Sry homologue murin de SRY.

\section{e) Le chromosome $X$.}

L'hypothèse du rôle du chromosome $\mathrm{X}$ dans la détermination testiculaire repose sur l'identification de cas familiaux de dysgénésie gonadique avec transmission liée à l'X et de cas sporadiques de réversion sexuelle chez des sujets SRY positif avec duplication du bras court de l'X [14]. Ogata, à partir de ces données suppose la présence d'un facteur de détermination testiculaire sur 1 X : TDF-X :

- TDF-X est soumis à l'inactivation de l'X.

- TDF-X pourrait être exprimé essentiellement, mais pas exclusivement dans les gonades.

- TDF-X pourrait fonctionner tôt dans le processus de détermination testiculaire.

- Rôle de TDF-X :

2 doses de TDF-X + SRY $\rightarrow$ Dysgénésie gonadique

1 ou 0 dose de TDF-X + SRY $\rightarrow$ détermination testiculaire

TDF-X agirait donc comme un répresseur de la détermination testiculaire, et SRY serait un répresseur de TDF.X en agissant dose pour dose, selon le schéma du tableau 1.

Le gène TDF-X a été localisé dans la région p21-3/21-1 du chromosome X. L'étude de sujets 46,XY ayant une réversion sexuelle a permis de définir une région "plus étroite» du chromosome X [DXS418-DXS319] de 10 à $20 \mathrm{Mb}$. Enfin, l'étude d'un patient avec une duplication vraie d'une région adjacente au locus de l'hypoplasie congénitale des surrénales, et d'un patient présentant une translocation d'une partie de l'X sur l'Y a permis de définir une région de $160 \mathrm{~kb}$ appelée GDXY dont le séquençage dira si elle contient un gène candidat pour être le TDF-X.

\section{CONCLUSION}

Les dysgénésies gonadiques présentent une hétérogénéité clinique, biologique, anatomique et génétique. Ces anomalies de la détermination testiculaire ont pour une grande part permis l'identification du gène de détermination testiculaire, SRY.

Cependant, si SRY semble vraiment avoir un rôle clé dans la détermination du sexe, il ne peut expliquer toute la pathologie de la détermination sexuelle comme l'absence de mutation du gène SRY dans la majorité des dysgénésies gonadiques, et comme les cas familiaux de dysgénésie gonadique avec mutation de SRY. Le processus de détermination testiculaire apparaît donc comme extrêmement complexe, faisant intervenir un ensemble de gènes en amont et en aval de SRY. Actuellement plusieurs candidats existent aussi bien sur des autosomes (chromosomes $1,9,10,11,17 \ldots)$ que sur le chromosome X (TDF-X).

La complexité du phénomène impose une démarche progressive. C'est l'expertise génétique moléculaire de nouveaux cas cliniques qui pourra permettre de mieux appréhender, au cours des prochaines années, les mécanismes moléculaires de la détermination sexuelle.

\section{REFERENCES}

1. BENNETT CP, DOCHERTY Z, ROBB SA, ET AL. : Deletion of $9 \mathrm{p}$ and sex reversal. J. Med. Genet, 1993, $30:$ 518-520. 


\begin{abstract}
$\begin{array}{ll}\text { Homme } & \text { Femme } \\ 46, X Y & 46, X X\end{array}$
SRY

inhibition de

l'expression de TDF-X

$\downarrow$

détermination testiculaire

\&

expression de TDF-X

détermination testiculaire

ㅁ

2. BERTA P, HAWKINS JR, SINCLAIR AH, ET AL. : Genetic evidence equating SRY and the testisdetermining factor. Nature, 1990, 348 : 448-450.

3. BERTA P, MORIN D, POULAT F, ET AL. : Molecular analysis of the sex determining region from the $\mathrm{Y}$ chromosome in two patients with Frasier syndrome. Horm. Res, 1992,37 : 103-106.
\end{abstract}

\author{
$46, \mathrm{XY}$ \\ duplication de l'Xp \\ ( 2 doses de TDF-X)

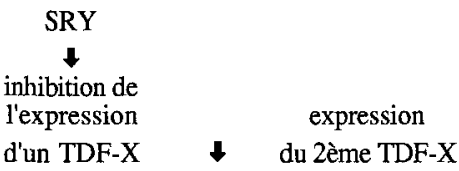

détermination testiculaire

\section{d'un TDF-X $\quad$ du 2ème TDF-X}

\author{
Homme \\ $46, \mathrm{XX}$ \\ mutation inactivatrice \\ de TDF-X \\ $\checkmark$
}

détermination testiculaire

\&
4. HAWKINS JR, TAYLOR A, BERTA P, ET AL :. Mutational analysis of SRY : nonsense and missense mutations in XY sex reversal. Hum. Genet, $1992,88: 471-474$.

5. HUFF DS, WU HY, SNYDER MMI, ET AL. : Evidence in favor of the mechanical (intrauterine torsion) theory over the endocrinopathy (cryptorchidism) theory in the pathogenesis of testicular agenesis. J. Urol, 1991, $146: 630-631$.

6. JÄGER RJ, ANVRET M, HALL K AND SCHERER G. : A human XY female with a frame shift mutation in the candidate testis-determining gene SRY. Nature, 1990, $348: 452-453$.

7. JÄGER RJ, HARLEY VR, PFEIFFER RA, GOODFELLOW PN AND SCHERER G. : A familial mutation in the Testis-Determining gene SRY shared by both sexes. Hum. Genet, 1992, $90: 350$ 355.

8. LOBACCARO JM, MEDLEJ R, BERTA P, ET AL. : PCR analysis and sequencing of the SRY sex determining gene in patients with bilateral congenital anorchia. Clin. Endocrinol, 1993, 38 : 197-201.

9. LOBACCARO JM AND SULTAN C. : La đifférenciation sexuelle normale : génétique et endocrinologie moléculaire. C. R. Soc. Biol, 1992, 186 : 314331 .
10. MCELREAVEY $\mathrm{K}$, VILAIN E, BOUCEKKINE C, ET AL. : XY sex reversal associated with a nonsense mutation in SRY. Genomics, 1992, $13: 838-840$.

11. MCELREAVY K, VILAIN E, ABBAS N, ET AL. : $\mathrm{XY}$ sex reversal associated with a deletion 5 ' to the SRY HMG box in the Testis-Determining region. Proc Natl Acad Sci USA, 1992, 89 : 1101611020.

12. MCGILLIVRAY MH AND MCGILLIVRAY B. : Hypogonadism and chromosomal disorders. In : Lifshits F eds. Pediatric Endocrinology. New York, Marcel Dekker, 1990, 377-412.

13. MENDEZ JP, ULLOA-AGUIRRE A, KOFMANALFARO S, ET AL. : Mixed gonadal dysgenesis: clinical, cytogenetic, endocrinological and histopathological findings in 16 patients. Am. J. Med. Genet, 1993, $46: 263-267$.

14. OGATA T, HAWKINS JR, TAYLOR A, ET AL. : Sex reversal in a child with a $46, \mathrm{Y}, \mathrm{Xp}+$ karyotype : support for the existence of a gene(s), located in distal Xp, involved in testis formation. J. Med. Genet, 1992, $29: 226-230$.

15. PELLETIER J, BRUENING W, KASHTAN CE, ET AL. : Germline mutations in Wilms' tumor suppressor gene are associated with abnormal urogenital development in Denys-Drash syndrome. Cell, 1991, $67: 437-447$.

16. POULAT F, MORIN D, KÖNIG A, ET AL. : Distinct molecular origins for Denys-Drash and Frasier syndromes. Hum. Genet, 1993, 91 : 285-286.

17. SULTAN C, LOBACCARO JM, MEDLEJ R, POULAT F AND BERTA P. : SRY and male sex determination. Horm. Res, $1991,36: 1-3$. 
18. TOMMERUP N, SCHEMPP W, MEINECKE P, ET AL. : Assignement of an autosomal sex reversal locus (SRA1) and campomelic dysplasia (CMPD1) to $17 \mathrm{q} 24.3-\mathrm{q} 25.1$. Nature Genet, $1993,4: \mathbf{1 7 0 - 1 7 4}$.

19. VILAIN E, JAUBERT F, FELLOUS M AND MCELREAVEY K. : Pathology of $46, \mathrm{XY}$ pure gonadal dysgenesis: absence of testis differentiation associated with mutations in the testis-determining factor. Differentiation, 1993, $52: 151-159$.

20. VILAIN E, MCELREAVEY K, VIDAUD M, RICHAUD F AND FELLOUS M. : L'analyse de SRY n'explique pas toute la pathologie du déterminisme du sexe. Ann. Endocrinol. (Paris), 1991, $52: 435-436$.

21. WILKIE AOM, CAMPBELL FM, DAUBENEY P, ET AL. : Complete and partial XY sex reversal associated with terminal deletion of $10 q$ - Report of 2 cases and literature review. Am. J. Med. Genet, $1993,46: 597-600$.

\section{ABSTRACT \\ Molecular genetic of testis dysgenesis \\ Ch. Sultan, S. Lumbroso, J.M. LOBACCARO}

Unité de Biochimie Endocrinienne du Développement et de la Reproduction, Hôpital Lapeyronie et INSERM U58, Montpellier - Endocrino-Gynécologie Pédiatriques, Service de Pédiatrie 1, Hôpital Arnaud de Villeneuve, Montpellier

The first step of male differentiation is the testis determination which is genetically controled. The key role of SRY gene is now established. However, a number of clinical and genetic data favoured the role of other genes taking place upstream or downstream SRY.

Most of 46,XX males possess a translocated SRY gene and thus develop testis, but SRY gene is not found in $10 \%$ of such patients. Likewise, the molecular study of $46, X Y$ females participated in the identification of SRY as testis determining factor, but $80 \%$ of $\mathrm{XY}$ gonadal dysgenesis are not explained by an abnormality of SRY gene.

Several clinical situations permitted to suspect the role of autosomal (chromosome $1,9,1017 \ldots)$ and $X$ chromosome loci in the pathology of sex determination. Some recent works concern, in particular, the testis determining factor of the $X$ chromosome (TDF-X) that could act as a repressor of the testis differentiation.

In conclusion, molecular mechanisms of sexual determination appear to be much complex, involving probably several genes in a pathway that remain to be elucidated.

Key words : sexual determination, SRY gene, $\mathrm{XX}$ males, testis dysgenesis 